\title{
Crank Cam Signal Generator Using NXP Model Based Design Toolbox
}

\author{
${ }^{1}$ Senol Gulgonul and ${ }^{2}$ Nedim Sozbir \\ ${ }^{1}$ Electronic Control Department, BMC Power Motor ve Kontrol Sistemleri, Istanbul, 34906, Turkey \\ ${ }^{2}$ Mechanical Engineering Department, Sakarya University, Sakarya, 54187, Turkey
}

\begin{abstract}
In this study, a cam-crank signal generator developed using Simulink and NXP Model Based Design Toolbox. Developed models compiled and flashed into NXP MPC5744P microcontroller. Generated cam and crank signals were measured using an oscilloscope for verification. Algorithm and Simulink models are describes in detail such that students and researchers can replicate and use in their studies using NXP or different microcontroller targets.
\end{abstract}

Key words: Crank, cam, model based design, NXP, diesel engine

\section{Introduction}

Four stroke diesel engines have four cycles; intake, compression, combustion and exhaust. Top dead center (TDC) is the point where piston reaches to the top point at compression cycle. Crankshaft rotates twice during these four cycle. Thus, crank angle has a range $0-720^{\circ}$ where $0^{\circ}$ is TDC point. Start of injection has a decisive effect on emission, fuel consumption and noise and plays an important role in optimizing engine performance characteristics. Fuel injection starts $0-8^{\circ}$ crank angle before TDC on a diesel engine. Camshaft controls exchange of gas during intake and exhaust phases. Camshaft rotates once during four cycle and has range of $0-360^{\circ}[1]$.

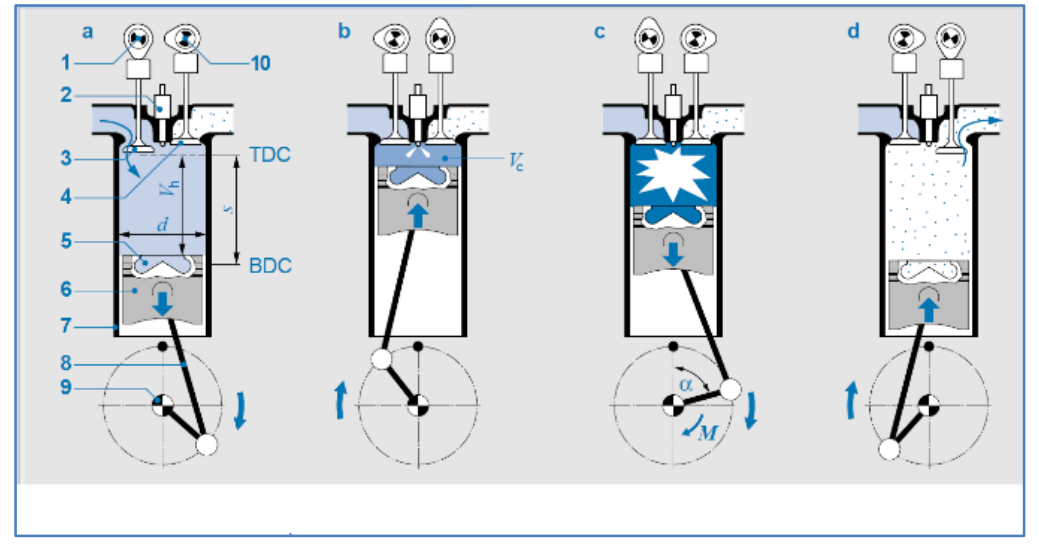

Figure 1. Four cycles of diesel engines; intake, compression, combustion and exhaust

*Corresponding author: Address: Faculty of Engineering, Department of Mechanical Engineering Sakarya University, 54187, Sakarya TURKEY. E-mail address: sozbir@sakarya.edu.tr, Phone: +902642955866 
Angles of camshaft and crankshaft are necessary for Engine Control Unit (ECU) to start injection fuel at proper position of the piston. Angles are measure through speed sensors, which are sensitive to distance to ferrous metal or magnetic materials. There are two type of speed sensors; variable reluctance sensors (VRS) and Hall-effect sensors. VRS sensors are passive, does not need a supply voltage and creates sinusoidal like output voltages from millivolts to hundred volts depending to speed of rotation and air gap (1-2mm) between sensor and metal. Hall-effect sensors re-quires a supply voltage from ECU and yields a clean square wave with fixed voltage level output which easy to process by ECU. Hall-effect sensor can also provide a second phase shifted output or directly provide a DC direction signal. Hall-effect sensors are able to detect zero speed and has to be used vehicle with start-stop functionality [2]. Hall-effect sensors simulated in this study for different camshaft and crankshaft tooth patterns.

Due to difficulties and cost of having a diesel engine at office environment, hardware in the loop (HIL), systems simulates the diesel engine during development of tests ECU. The main component of a HIL system is simulation of cam and crank speed sensor signals varying with engine rpm. Commercial HIL systems (e.g. dSpace, ETAS) are modular to cover whole functionality of engines, sensors and actuators, not limited to crank and cam signals.

VRS type crank and hall-effect type cam speed sensors were simulated in a study where Simulink models compiled and downloaded on a target PC running on xPC Real-Time Kernel [3]. NI-9068 CompactRIO Controller, Arduino and TMS320C31 were programmed to simulate cam and crank signals $[4,9,11]$. Some NXP microcontrollers with eTPU functionality are able to simulate cam and crank signals [10].

\section{Model Development}

\subsection{Crank Model}

Crankshaft is the main source of torque at diesel engines, driven by pistons and completes $720^{\circ}$ rotation during four strokes. Speed sensor has to be mounted against tooth or halls on the flywheel. Crankshaft flywheel has generally 60-2 or 36-1 tooth pattern [5,8]. Missing teeth required to define zero crank angle. The crank angle reference point; zero degree is defined as the first rising or falling edge after the gap created by missing teeth [6].

Periodic interrupt timer (PIT) calls crank function at an interval inversely proportional to engine rpm. PIT period will be half of the period of on tooth due to upper and lower sides of a tooth. Period of PIT (seconds) can be calculated as:

$$
\mathrm{p}=\frac{60}{2 \times n \times r}
$$

where $\mathrm{n}$ denotes the number of tooth, $\mathrm{r}$ is engine speed and multiplier 2 is ratio between PIT period and tooth period. PIT periods of 500-100 microseconds are suitable for diesel engines at 1000$5000 \mathrm{rpm}$. 


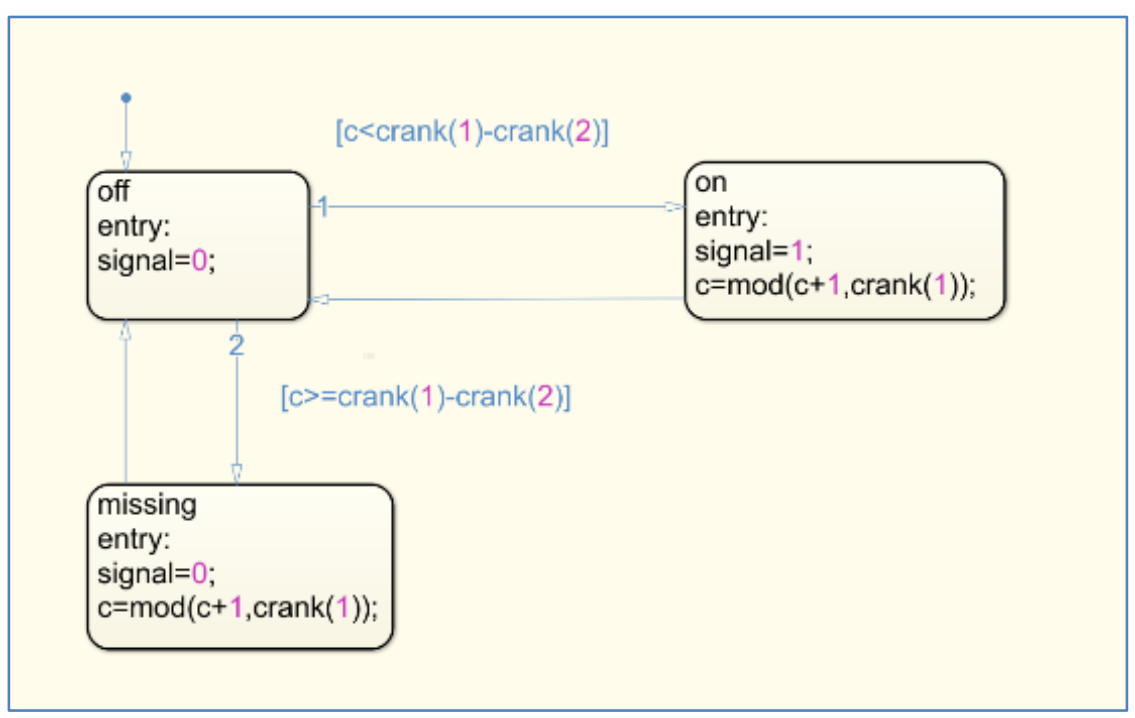

Figure 2. Crank signal stateflow chart

Number of tooth on crankshaft is an array at Simulink model workspace. The first value is total number of tooth and the second is number of missing teeth, e.g. crank $=[60,2]$. Crank signal is created using a stateflow chart (Figure 2.). Chart changes its state at each PIT interval. A counter c counts number of tooth with modulus of total number of tooth (e.g. 60). If counted number of tooth less than total number of tooth minus number of missing teeth (e.g. 58) signal value becomes 1. If this condition does not satisfied signal value returns to zero (Figure 3.).

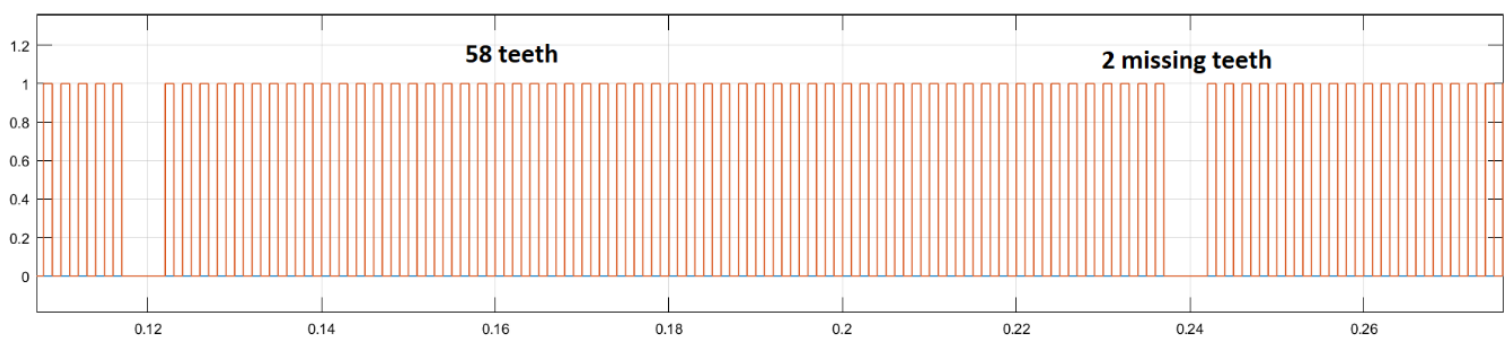

Figure 3. Crank signal created by stateflow chart

\subsection{Cam Model}

While crankshaft rotates twice during four strokes of diesel engine, camshaft rotates one tour. Since crankshaft rotates twice, it is not possible to detect with only missing tooth reference whether the crankshaft is in the first or second tour. Thus, camshaft reference is required additionally as a second reference point.

Cam signal is again generated using Simulink stateflow charts called at each interval of PIT timer. Cam tooth configuration is defined as an array with number pairs of first and second crank tooth numbers where cam tooth coincides. This method allows defining various cam configurations 
including single tooth, half moon, $6+1$ etc [5]. Cam tooth configuration can be defined as an array of cam=[103 104] where cam tooth must occur during 103-104 crank teeth, keeping in mind that 2 crank revolutions will sweep $2 \times 60=120$ crank teeth. Width of the cam tooth is not fixed and can be changed according to the required application. $6+1$ cam tooth pattern can be define in array:

$$
c a m=[13153335535573759395108110113115]
$$

Half moon or single tooth configurations has only one cam tooth. Edges of this tooth shall be outside of missing tooth region in order to decide the first or second rotation of the crankshaft. Crankshaft first half starts after the camshaft refer-ence point (Figure 4.).

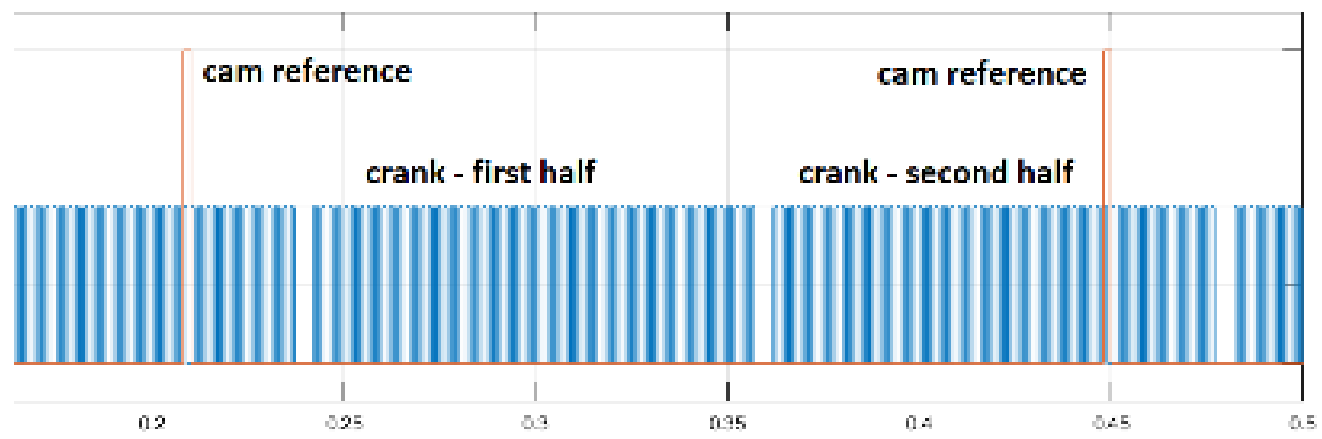

Figure 4. Single tooth cam and 60-2 crank patterns

$6+1$ camshaft tooth configuration has advantage of providing crank angle in case of crank speed sensor failures. Since camshaft rotates only once during four strokes of a diesel engine, sweeping $0-360^{\circ}$, a reference point to define zero angle and additional reference points to measure angles provides all the information needed. Six teeth is equally divides $360^{\circ}$ into $60^{\circ}$ parts. Additional +1 tooth defines where zero cam angle starts. Zero cam angle for $6+1$ camshaft is the first tooth after +1 additional tooth. First half of the crank comes just after the +1 cam tooth (Figure 5.) [5].

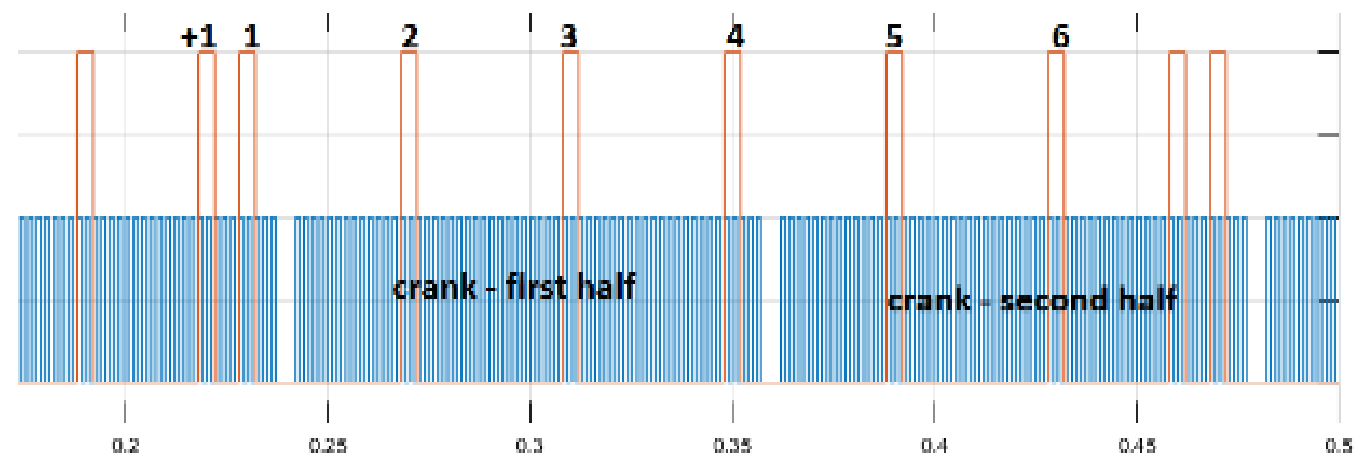

Figure 5. 6+1 cam and 60-2 crank patterns 
Cam stateflow model is similar to crank model. A counter c counts PIT intervals. If the calculated tooth number is between pairs of array, cam signal becomes positive. The counter $\mathrm{i}$ is starting from zero, increments index and selects next pairs of cam array.

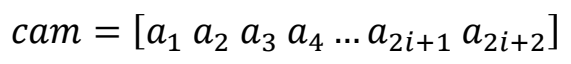

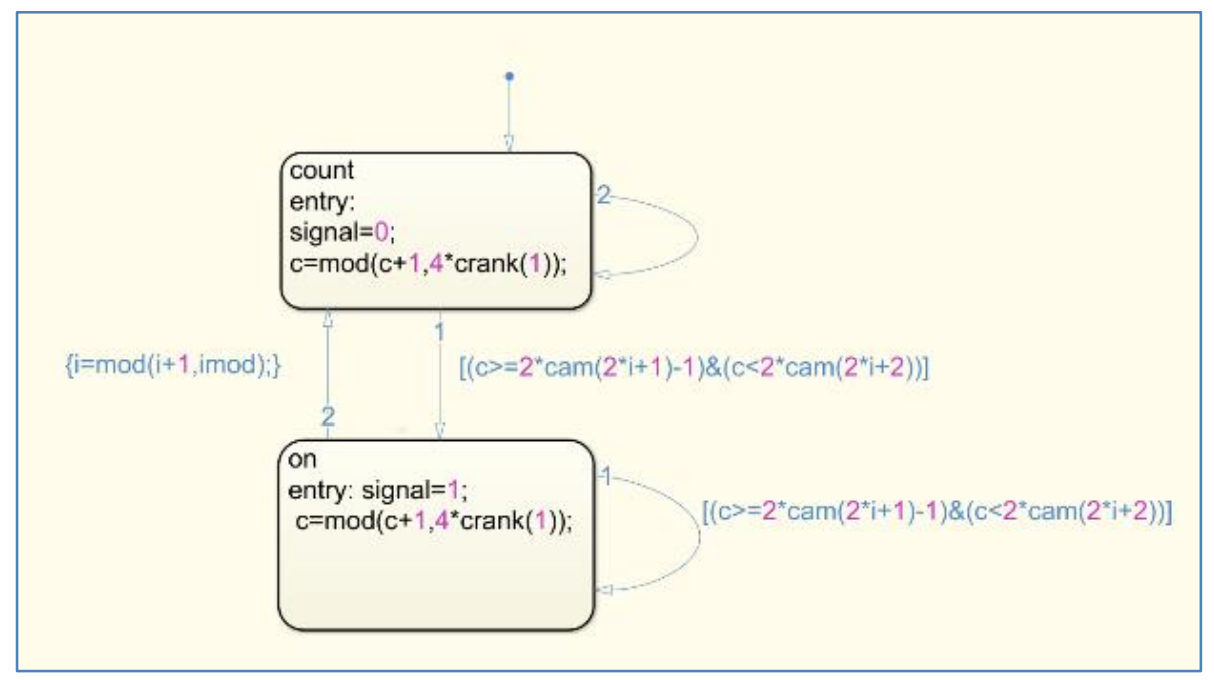

Figure 6. Cam stateflow model

\subsection{Complete Model}

Model based design toolbox version 3.0 configure 5744p configuration parameters block. Freemaster block is necessary during development debugging output parameters and signals cam_on and crank_on. PIT block is an interrupt timer calls CAM_CRANK function at every defined microseconds (Figure 7.). 


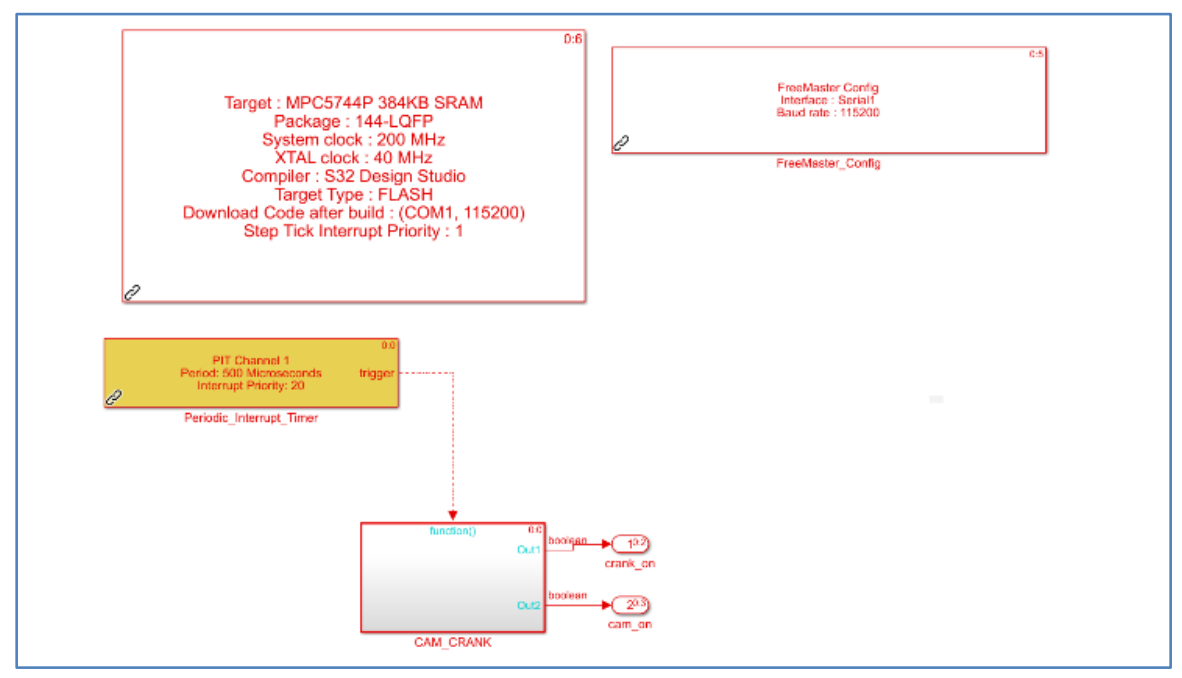

Figure 7. Complete view of cam and crank signal generator model

Function call CAM_CRANK has two stateflow charts, which were described in previous sections. There is data type converter to convert double value to boolean for GPIO inputs. PDT5 and PTD6 digital GPIO output provides cam and crank signals (Figure 8.).

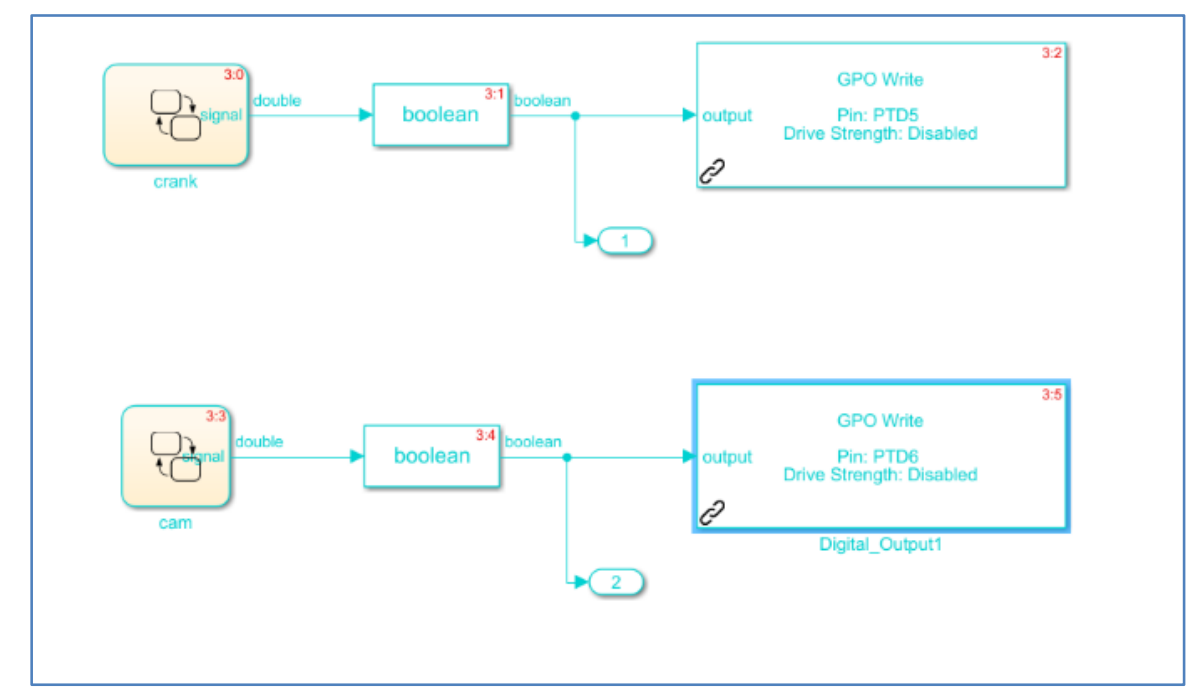

Figure 8. Inside of function call block

\section{Results}

NXP MPC5744p development kit is a tool engineering for automotive and motor control applicationsi based on 32 bit Power Architecture provides more than 100 GPIOs plus CAN, LIN, FlexRay communication interfaces [7]. NXP's Model Based Design Toolbox (MBDT) provides Simulink libraries for configuring and generating the software code necessary to execute complex 
control applications. NXP's MBDT supports MPC5744p target. Thus, MPC5744p was selected for cam crank signal generator application.

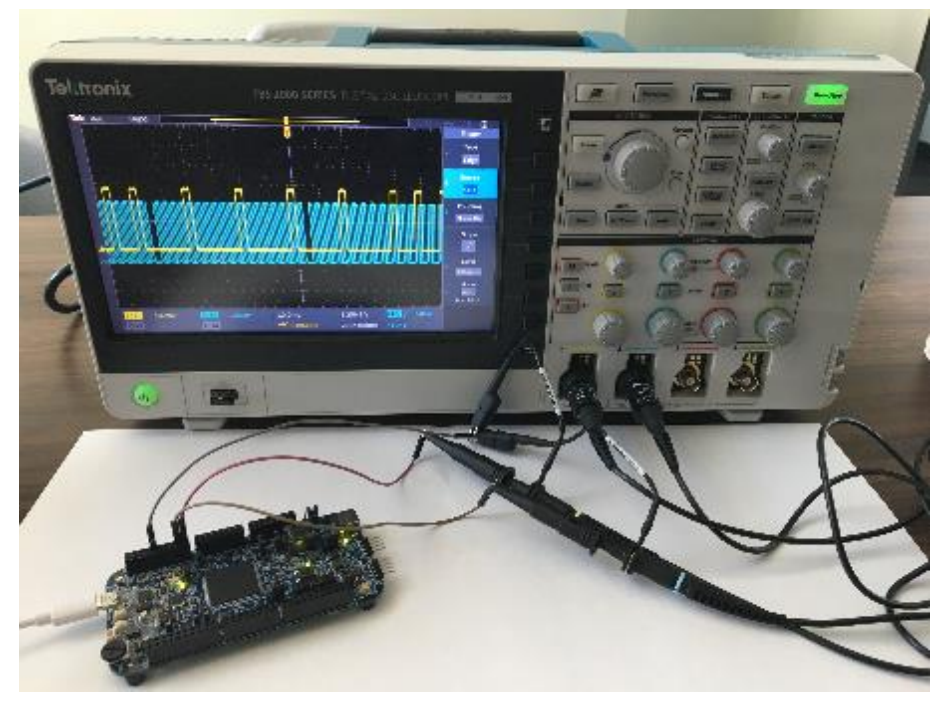

Figure 9. Test setup

MPC5744p is powered through USB port of a notebook. PTD5 and PTD6 digital GPIO output ports are connected to channels $\mathrm{CH} 1$ and $\mathrm{CH} 2$ of Tektronix TBS 2000 series digital oscilloscope respectively. PIT interval is set to 500 microseconds to represent $1000 \mathrm{rpm}$ engine speed. Single tooth configuration has cam tooth at 103-104 of crank teeth as show on Figure 10

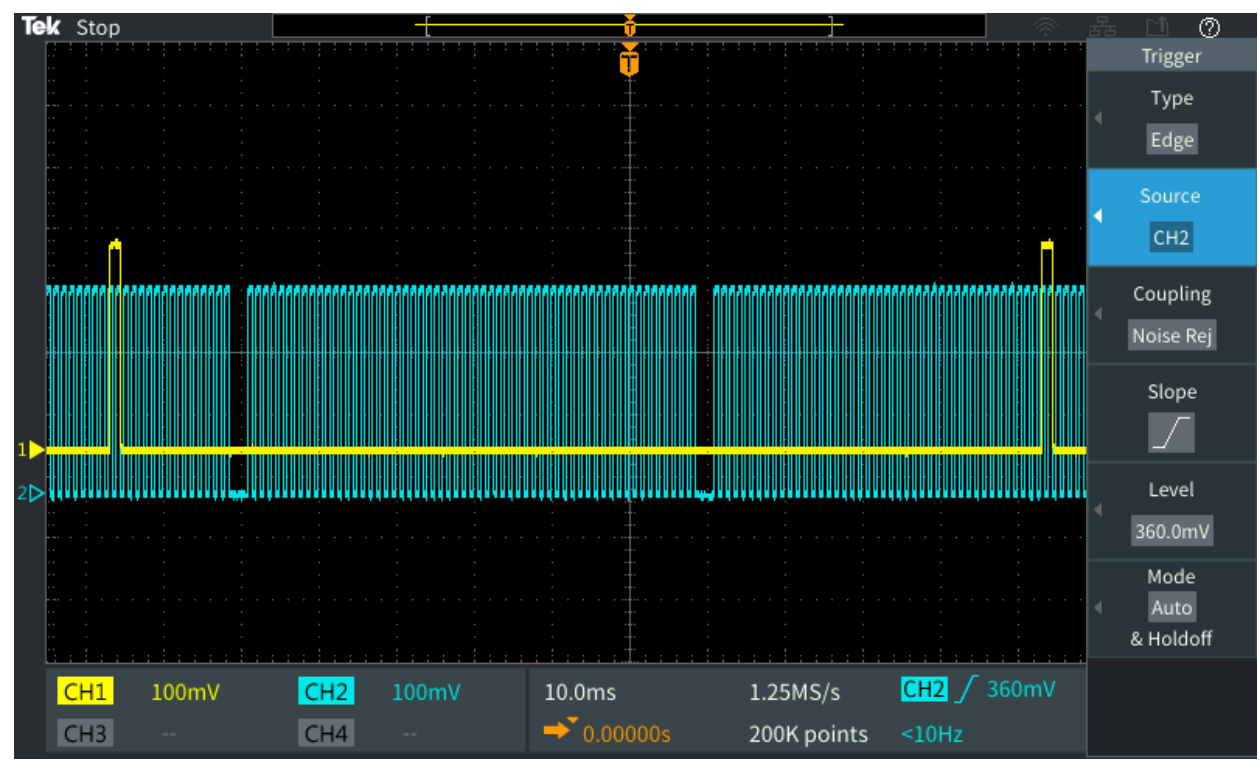

Figure 10. Single tooth cam and 60-2 crank signal patterns 
$6+1$ tooth configurations has the same scene of simulation as captured on oscilloscope (Figure 11.). Signal generator was able to generate cam and crank signals as expected at simulation outputs shown at Figure 4. and Figure 5.

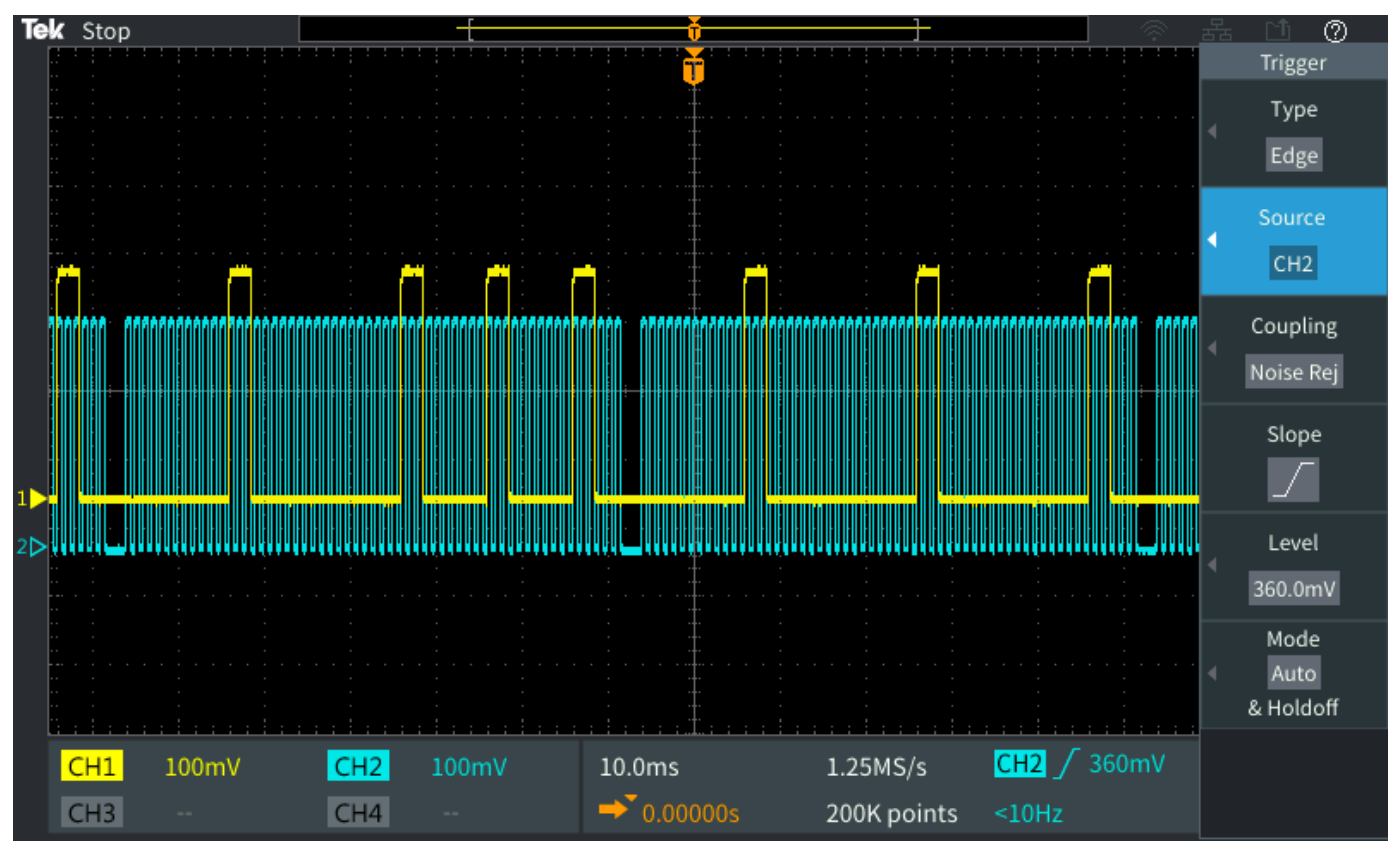

Figure 21. 6+1 cam and 60-2 crank signal patterns

\section{Conclusions}

Model and implementation of a developed crank and cam signal generator has been presented in this study. Model of cam and crank signals were developed in Simulink environment. The model compiled using Simulink coder and flashed into target microcontroller NXP's MPC5744p development kit. Output ports of the MPC5744p kit connected to oscilloscope to observe and measure real signals. Details of the model described in this study so that students or researchers can implement the same model to their preferred microcontrollers to simulate cam and crank signals generated on a diesel engine. Model based design has flexibility to compile and embed the same models to different target microcontrollers (Arduino, STM etc) 


\section{References}

[1] Bauer H. Diesel Engine Management. Robert Bosch GmbH. 2003.

[2] Infineon Technologies AG. TLE 4922 Cost Efficient Hall Sensor User Manual. Infineon Technologies AG. 2017.

[3] Lian H, Cai Z, Luo W, Zeng C and Fang D., "Matlab/Simulink based crankshaft and camshaft signal simulation model for SI engine," 35th Chinese Control Conference (CCC), Chengdu, 2016, pp. 8896-8901.

[4] Drosescu R. P Virtual engine management simulator for educational purposes. IOP Conf. Ser. 2017: Mater. Sci. Eng. 252012099.

[5] http://mcs.woodward.com/content/motohawk/Documentation/MotoHawk2015bSP0/HTML/ MotoHawk_lib_Blocks/EncoderDefinition.html

[6] Mike P. AN2897 Using the eTPU Angle Clock. Freescale Semiconductor Application Note. 2004.

[7] NXP Semiconductors. MPC5744P Data Sheet. 2017.

[8] http://support.openecu.com/doc_user/openecu_user_guide_simulink_chunk/pan_crank_wheel _config.html

[9] Hermansson E, Serti T. Speed Sensor Simulator Box. Bachelor thesis, Department of Signals and Systems Chalmers University of Technology, Gothenburg, Sweden. 2015.

[10] Brejl M. AN3801 Using the eTPU Angle Clock. Freescale Semiconductor Application Note. 2009.

[11] Hanselmann H. DSP-Based Automotive Sensor Signal Generation for Hardware-in-the-Loop Simulation. SAE Transactions, 1994. 103, 85-91. 